\title{
Effects of ionic and non-ionic micelles on rate of hydroxide ion-catalyzed hydrolysis of securinine
}

\begin{abstract}
The effects of micelles of cetyltrimethylammonium bromide (CTABr), tetradecyltrimethylammonium bromide (TTABr), sodium dodecyl sulfate (SDS) and polyoxyethylene 10 lauryl ether (C12E10) on the rates of alkaline hydrolysis of securinine were studied at a constant [i $\mathrm{OH}](0.05 \mathrm{M})$. An increase in the total concentrations of CTABr, TTABr, SDS and C12E10 from 0.0 to $0.2 \mathrm{M}$ causes a decrease in the observed pseudo-firstorder rate constants (kobs) by factors of ca 2.5, 3, 7 and 4, respectively. The observed data are explained in terms of pseudophase and pseudophase ion-exchange (PIE) models of micelle. The binding constants, KS, of securinine with SDS, C12E10, CTABr and TTABr micelles are 32.4, 14.8, 22.1, and 9.1 Mī 1, respectively. The magnitudes of the second-order rate constants, $\mathrm{kM}$, for the reactions in the micellar pseudophase are negligible compared with the corresponding rate constant, $\mathrm{kW}$, for the reaction occurring in the aqueous pseudophase for CTABr, TTABr, SDS and C12E10.
\end{abstract}

Keyword: Alkaline hydrolysis; Anionic micelle; Cationic micelles; Kinetics; Non-ionic micelle; Securinine 